PAPER:

LMU-TPW 92-05

Mannheimer Manuskripte 141

May 1992

\title{
String Branchings on Complex Tori and Algebraic Representations of Generalized Krichever - Novikov Algebras
}

\author{
Andreas RufFing ${ }^{1,2}$, \\ Thomas Deck ${ }^{3}$, \\ MARTin SCHLiCHENMAIER ${ }^{3}$
}

\footnotetext{
${ }^{1}$ Sektion Physik der Universität München, Theresienstraße 37, D-W-8000 München 2, Germany

${ }^{2}$ Institut für Theoretische Physik, Universität Karlsruhe, D-W-7500 Karlsruhe 1, Germany

${ }^{3}$ Fakultät für Mathematik und Informatik, Universität Mannheim, A5, Postfach 103462,
} D-W-6800 Mannheim 1, Germany,

\begin{abstract}
The propagation differential for bosonic strings on a complex torus with three symmetric punctures is investigated. We study deformation aspects between two point and three point differentials as well as the behaviour of the corresponding KricheverNovikov algebras. The structure constants are calculated and from this we derive a central extension of the Krichever-Novikov algebras by means of $b-c$ systems. The defining cocycle for this central extension deforms to the well known Virasoro cocycle for certain kinds of degenerations of the torus.
\end{abstract}

AMS subject classification (1991). 17B66, 17B90, 14H52, 30F30, 81T40 


\section{Introduction}

String theory and conformal field theories were starting points for the investigation of Lie algebras of meromorphic vector fields on Riemann surfaces in the context of mathematical physics. In 1987 Krichever and Novikov [11] studied (beside other objects) algebras of meromorphic vector fields with poles only at fixed points $P_{+}, P_{-}$(called markings or punctures) on a compact Riemann surface $X$, as well as central extensions of such algebras, see also [14]. These algebras are nowadays called Krichever-Novikov algebras (KN algebras).

The generalization of their concept for more than two markings of a surface was given by Dick [4-8] and Schlichenmaier [15-18]. They studied algebras of meromorphic vector fields on a genus $g$ Riemann surface which have poles only at a finite number of fixed points. For related work see also [9]. In $[2,3]$ it was shown by Deck that a special choice of two punctures on a torus provides simple structure constants. They only contain the parameters of the Weierstraß $\wp$-function

$$
e_{1}:=\wp\left(\frac{1}{2}\right), \quad e_{2}:=\wp\left(\frac{1}{2}+\frac{\tau}{2}\right), \quad e_{3}:=\wp\left(\frac{\tau}{2}\right)
$$

Here $\tau$ denotes the normalized period of the torus. This fact allows one to consider deformations of the analytic structure in terms of $e_{1}, e_{2}, e_{3}$. In order to obtain similar results on a complex torus with three punctures (markings) and in order to study some further aspects of deformation (concerning the number of punctures) let us repeat some basic definitions.

Let $X$ be a compact Riemann surface of genus $g$ with $N$ fixed points $(N \geq 2)$. We denote the set of these points with $S$. Let $S$ split into two nonempty disjoint subsets $E$ and $O, S=E \cup O$. $E$ is called the set of "in-points" and $O$ the set of "out-points". Let $\omega$ be the unique meromorphic differential with only imaginary periods, holomorphic on $X \backslash S$ with poles of order 1 at $S$ and with the residues

$$
\operatorname{res}_{P}(\omega)=+\frac{1}{\# E}, \quad P \in E, \quad \text { and } \quad \operatorname{res}_{Q}(\omega)=-\frac{1}{\# O}, \quad Q \in O .
$$

In the following we restrict our investigations to $g=1$ (i.e. to the case of complex tori) and the special cases of one in-point $P_{1}$ and two out-points $Q_{1}, Q_{2}$. The well known case of two points will appear as a degenerated three point case. The generalizations to more than 3 points are discussed in [13].

We choose a point $R$ on $X \backslash S$. Due to the above properties the function

$$
t(P):=\operatorname{Re} \int_{R}^{P} \omega
$$


is well defined and harmonic on $X \backslash S$. The level lines $C_{u}:=\{P \in X \mid t(P)=u\}$ for $u \in \mathbb{R}$ define a global fibration of the surface $X \backslash S$ interpreted as the "position" of the string(s) at "time" $u$. For large negative $u$ these lines are small circles around the points in $E$ and for large positive $u$ they are small circles around the points in $O$. So the strings "travel" from the points with positive residues to those with negative residues. They are called "ingoing" strings at $E$ and "outgoing" strings at $O$. At the points were $\omega$ has a zero the level lines (strings) split or rejoin. Because of this physical interpretation $\omega$ is called propagation differential or differential of the time development. A physical realization of the KN algebra (with central extensions) is given by the commutation relations of the components of the energy-momentum tensor which belong to a string moving in Minkowski space [11].

\section{The propagation differential}

We consider a torus $T=\mathbb{C} / L$ with $L:=\{z \in \mathbb{C} \mid z=m+n \tau, \quad m, n \in \mathbb{Z}\}$ and $\tau \in \mathbb{C}, \operatorname{Im} \tau>0$.

We need the following facts about the Weierstraß $\wp$-function [10]:

$\wp$ is an even meromorphic doubly periodic function (i.e. $\wp(z+w)=\wp(z), \forall w \in L)$ with a second order pole only in the lattice points and holomorphic elsewhere with Laurent expansion $\wp(z)=z^{-2}+\cdots$. By the covering map $\pi: \mathbb{C} \rightarrow \mathbb{C} / L$ we have a holomorphic 1 -form $d z$ on the torus $T$.

As markings we choose the following points

$$
P_{1}=0 \quad \bmod L, \quad Q_{1}=1 / 2+q \bmod L, \quad Q_{2}=1 / 2-q \bmod L,
$$

with $q \neq 0,1 / 2 \bmod L$.

We construct now a differential $\omega$ with the properties

(1) $\omega$ is holomorphic on $T \backslash\left\{P_{1}, Q_{1}, Q_{2}\right\}$,

(2) $\operatorname{res}_{P_{1}}(\omega)=+1$ and $\operatorname{res}_{Q_{i}}(\omega)=-1 / 2, i=1,2$,

(3) $\omega$ has only imaginary periods.

From considerations about the (probable) zeros of $\omega$ (the set $\{1 / 2, \tau / 2,(1+\tau) / 2\}$ ) we found the following result

Proposition. The propagation differential with respect to the punctures (4) is given by

$$
\omega_{q}(z)=\widehat{\omega}_{q}(z) d z=-\frac{1}{2} \frac{\wp^{\prime}(z)}{\wp(z)-\wp(1 / 2+q)} d z
$$

Proof. The singularities of $\omega$ (all of first order) follow from the facts about $\wp(z)$ as well as the property $\operatorname{res}_{0}(\omega)=1$. 
Also we have $\wp(z)=-\wp(-z)$ and this yields together with $\sum_{Q \in T} \operatorname{res}_{Q}(\omega)=0$ (residue theorem) $\operatorname{res}_{1 / 2+q}(\omega)=\operatorname{res}_{1 / 2-q}(\omega)=-1 / 2$ and hence the property $(2)$.

The final requirement (3) follows from the fact that $\omega$ is antisymmetric in the argument with respect to the points $0,1 / 2,(1+\tau) / 2$ and $\tau / 2$ and has purely real residues.

Note, in the proof above we used $\operatorname{res}_{z} \bmod L=\operatorname{res}_{z}$.

\section{Degenerated case and calculation of the separation time}

If we now set $q=0$ we will find a holomorphic differential on a 2-punctured torus:

$$
\omega_{0}(z)=\widehat{\omega}_{0}(z) d z=-\frac{1}{2} \frac{\wp^{\prime}(z)}{\wp(z)-e_{1}} d z .
$$

Obviously we have $\operatorname{res}_{0}\left(\omega_{0}\right)=-\operatorname{res}_{1 / 2}\left(\omega_{0}\right)=+1$. All other required properties of a propagation differential are conserved and so $\omega_{0}$ describes a process of pure generation and annihilation of a string-pairing. In the limit $q=0$ the two poles of $\omega_{q}$ for outgoing strings coincide in $1 / 2$. This case has been studied in [3] and is a special case in our prescription. The differential $\omega_{q}$ has a big advantage: It's real part can be integrated at once because it has the structure of a logarithmic differential. If we integrate between the two zeros $\tau / 2$ and $(1+\tau) / 2$ of $\omega_{q}$ we find

$$
(\Delta t)_{q}:=\operatorname{Re} \int_{\tau / 2}^{(1+\tau) / 2} \omega_{q}=\frac{1}{2} \ln \left|\frac{e_{3}-\wp(1 / 2+q)}{e_{2}-\wp(1 / 2+q)}\right|
$$

and

$$
(\Delta t)_{0}=\frac{1}{2} \ln \left|\frac{e_{3}-e_{1}}{e_{2}-e_{1}}\right|
$$

as an obvious limit for $q \rightarrow 0$.

From the physical background we call $(\Delta t)_{q}$ separation time. We only give an interpretation of $(7)$. Here we find $(\Delta t)_{0}=-\frac{1}{2} \ln |\mu|$ where the parameter $\mu:=\left(e_{2}-e_{1}\right)\left(e_{3}-e_{1}\right)^{-1}$ is directly related to the algebraic equation of $\wp, \wp^{\prime}$.

More precisely, $\mu$ is the moduli parameter for tori with level 2 structure, i.e. for tori with fixed 2-torsion points. If we introduce the principal congruence subgroup of level 2

$$
\Gamma(2):=\left\{A \in \mathrm{SL}(2, \mathbb{Z}) \mid A \equiv\left(\begin{array}{ll}
1 & 0 \\
0 & 1
\end{array}\right) \quad \bmod 2\right\}
$$

of the full modular group $\mathrm{SL}(2, \mathbb{Z})$ then the moduli space of tori with level 2 structure is the quotient of the upper half-space $\mathcal{H}:=\{z \in \mathbb{C} \mid \operatorname{Im} z>0\}$ with respect to the action of $\Gamma(2)$ by fractional linear transformation. In other words

$$
z \sim z^{\prime} \Longleftrightarrow z^{\prime}=\frac{a z+b}{c z+d}, \quad\left(\begin{array}{ll}
a & b \\
c & d
\end{array}\right) \in \Gamma(2) .
$$


It is well known (see [12, App.7]) that $|\mu|=1$ iff the lattice parameter $\tau$ is equivalent to a $\tau^{\prime}$ under the relation (8) with $\operatorname{Re} \tau^{\prime}= \pm 1 / 2$.

\section{The corresponding Lie algebra of vector fields}

The KN algebra is given by those meromorphic vector fields which are holomorphic on $T \backslash S$. There is a natural identification of meromorphic functions and meromorphic vector fields on a complex torus by the correspondence $f(z) \mapsto f(z) \frac{d}{d z}$. Let us therefore consider an infinite set $\mathcal{A}=\left\{A_{k}\right\}_{k \in \mathbb{Z}}$ of functions. The elements are given by

$$
\begin{aligned}
& A_{n}(z):=\exp \left(n \int_{\lambda}^{z} \omega_{q}\right)=a_{n}(\lambda)(\wp(z)-\wp(1 / 2+q))^{-n / 2}, \quad n \in 2 \mathbb{Z}, \\
& A_{\alpha}(z):=\widehat{\omega}_{q}(z) A_{\alpha+1}(z), \quad \alpha \in 2 \mathbb{Z}+1
\end{aligned}
$$

We choose $\lambda \in \mathbb{C}$ such that $a_{2}(\lambda)=1$ and hence all $a_{n}(\lambda)=1$. Note that $A_{n}=\left(A_{2}\right)^{n / 2}$. It is worth-while mentioning that all these functions are given in terms of the propagation differential $\omega_{q}$. Obviously the $A_{n}$ are even functions whereas the $A_{\alpha}$ are odd functions. From now on we denote even indices with $m, n$ and odd ones with $\alpha, \beta$. For convenience let us introduce an order triple of a function $f$ :

$$
f^{*}:=\left(\operatorname{ord}_{0} f, \operatorname{ord}_{1 / 2+q} f, \operatorname{ord}_{1 / 2-q} f\right) \in \mathbb{Z}^{3}
$$

We then have

$$
A_{n}^{*}=(n,-n / 2,-n / 2) \quad \text { and } \quad A_{\alpha}^{*}=(\alpha,(-\alpha-3) / 2,(-\alpha-3) / 2) .
$$

Considering the $\operatorname{ord}_{0}$ entry it is evident that the set $\mathcal{A}$ is linearly independent over $\mathbb{C}$.

Proposition. A basis of meromorphic functions on $T$ which are holomorphic on $T \backslash S$ is given by the set $\mathcal{A}$.

Proof. There are at least two ways to show that $\mathcal{A}$ represents a basis of vector fields. Each way gives insights which will we useful later on.

1. One can show that there exists linear combinations $E_{1}, E_{2}, E_{3}, E_{4}$ by elements of $\mathcal{A}$ so that $E_{1}^{*}=(-1,-1,0), \quad E_{2}^{*}=(0,-1,-1), \quad E_{3}^{*}=(0,0,2), \quad E_{4}^{*}=(2,-1,-1)$. For instance such an element $E_{1}$ can be obtained as a certain linear combination of $A_{2}$ and $A_{-1}$ by looking at their analytic behaviour near $1 / 2 \pm q$. It can be shown that any function $f$ on the torus with markings in $S$ can be algebraically combined by $E_{1}, E_{2}, E_{3}, E_{4}$ and $A_{0}$. We have

$$
A_{i} A_{j}=A_{j} A_{i}=A_{i+j} \quad \text { for } \quad i \in 2 \mathbb{Z}, j \in \mathbb{Z}
$$


and $\left(\widehat{\omega}_{q}^{2}\right)^{*}=(-2,-2,-2)$. As $\widehat{\omega}_{q}, A_{-2}, A_{0}, A_{2}, A_{4}$ are even function one can show using (12) that

$$
\widehat{\omega}_{q}^{2}=\lambda_{1} A_{-2}+\lambda_{2} A_{0}+\lambda_{3} A_{2}+\lambda_{4} A_{4}, \quad \text { with } \quad \lambda_{1}, \lambda_{2}, \lambda_{3}, \lambda_{4} \in \mathbb{C}
$$

Hence, we obtain with the same values for $\lambda_{i}$

$$
A_{i} A_{j}=\lambda_{1} A_{i+j}+\lambda_{2} A_{i+j+2}+\lambda_{3} A_{i+j+4}+\lambda_{4} A_{i+j+6} \quad \text { for } \quad i, j \in 2 \mathbb{Z}+1
$$

Now one can easily see that any $f$ is a linear combination of elements of $A$.

2. Because $A_{n}$ is even and $A_{\alpha}$ is odd there are linear combinations $B_{k}=\gamma A_{2 k}+\delta A_{2 k-3}, \quad C_{k}=\gamma^{\prime} A_{2 k}+\delta^{\prime} A_{2 k-3} \quad$ with order triple $B_{k}^{*}=(2 k-3,-k, b)$, $C_{k}^{*}=(2 k-3, c,-k)$ and $b, c>-k$. By a simple elimination argument concerning the pole orders of an arbitrary function $f$ ( of the prescribed type) it is verified that $f$ is a linear combination of the $A_{k}, B_{k}, C_{k}$ and consequently a linear combination of the set $\mathcal{A}$ alone.

The next step is the computation of the Lie algebra of vector fields which are given by $l_{k}(z):=A_{k}(z) \frac{d}{d z}$ under Lie bracket. From (9) and (10) we find

$$
\begin{aligned}
& {\left[l_{n}, l_{m}\right](z)=(m-n) l_{m+n-1}(z)} \\
& {\left[l_{\alpha}, l_{\beta}\right](z)=(\beta-\alpha) \widehat{\omega}_{q}^{2}(z) l_{\alpha+\beta+1}(z)} \\
& {\left[l_{\alpha}, l_{n}\right](z)=\left((n-\alpha+1) \widehat{\omega}_{q}^{2}(z)+\widehat{\omega}_{q}^{\prime}(z)\right) l_{n+\alpha+1}(z)}
\end{aligned}
$$

It is an interesting fact that the whole structure of the algebra is encoded in $\omega_{q}[13]$. The element $\widehat{\omega}_{q}^{\prime}$ is (like $\widehat{\omega}_{q}^{2}$ ) an even functions hence a linear combination of the even elements $A_{n}$. To obtain its explicit representation we use $A_{n}^{\prime}(z)=n \widehat{\omega}_{q}(z) A_{n}(z)$. Using this and the representation (14) for $\widehat{\omega}_{q}^{2}$ we can deduce from $\left(\widehat{\omega}_{q}^{2}\right)^{\prime}(z)=2 \widehat{\omega}_{q}(z) \widehat{\omega}_{q}^{\prime}(z)$

$$
\widehat{\omega}_{q}^{\prime}(z)=-2 \lambda_{1} A_{-2}(z)+2 \lambda_{3} A_{2}(z)+4 \lambda_{4} A_{4}(z)
$$

Together with property (13) the structure coefficients of the algebra immediately follow from (16)-(18). The remaining task is to find the coefficients $\lambda_{1}, \lambda_{2}, \lambda_{3}, \lambda_{4}$. But this can be done in an algebraic way:

Substituting the Weierstraß relation $\wp^{\prime}=4\left(\wp-e_{1}\right)\left(\wp-e_{2}\right)\left(\wp-e_{3}\right)$ into

$$
\widehat{\omega}_{q}^{2}(z)=\frac{1}{4} \frac{\wp^{\prime 2}(z)}{(\wp(z)-\wp(1 / 2+q))^{2}}
$$

as well as the expression (9) into (14) we find a polynomial identity in the variable $X:=\wp(z)$ from which we can recognize the coefficients $\lambda_{1}, \lambda_{2}, \lambda_{3}, \lambda_{4}$. The computation 
finally yields the required generalized Krichever-Novikov algebra:

$$
\begin{aligned}
{\left[l_{n}, l_{m}\right]=} & (m-n) l_{n+m-1}, \\
{\left[l_{\alpha}, l_{\beta}\right]=} & (\beta-\alpha)\left(l_{\alpha+\beta-1}+3 \wp(1 / 2+q) l_{\alpha+\beta+1}+\right. \\
& \left(3 \wp^{2}(1 / 2+q)-\left(e_{2}^{2}+e_{2} e_{3}+e_{3}^{2}\right)\right) l_{\alpha+\beta+3}+ \\
& \left.1 / 4 \wp^{\prime 2}(1 / 2+q) l_{\alpha+\beta+5}\right) \\
{\left[l_{\alpha}, l_{n}\right]=} & (n-\alpha) l_{\alpha+n-1}+(n-\alpha-1) 3 \wp(1 / 2+q) l_{\alpha+\beta+1}+ \\
& (n-\alpha-2)\left(3 \wp^{2}(1 / 2+q)-\left(e_{2}^{2}+e_{2} e_{3}+e_{3}^{2}\right)\right) l_{\alpha+n+3}+ \\
& (n-\alpha-3) 1 / 4 \wp^{\prime 2}(1 / 2+q) l_{\alpha+n+5} .
\end{aligned}
$$

Indeed the case $q=0$ coincides with that given in [3]. In this sense we have another aspect of deformation in the family of $\mathrm{KN}$ algebras. The two point algebra $(q=0)$ is obtained by taking the limit $q \rightarrow 0$ of the three point algebra:

$$
\begin{aligned}
{\left[l_{n}, l_{m}\right]=} & (m-n) l_{n+m-1}, \\
{\left[l_{\alpha}, l_{\beta}\right]=} & (\beta-\alpha)\left(l_{\alpha+\beta-1}+3 e_{1} l_{\alpha+\beta+1}+\left(e_{1}-e_{2}\right)\left(e_{1}-e_{3}\right) l_{\alpha+\beta+3}\right), \\
{\left[l_{\alpha}, l_{n}\right]=} & (n-\alpha) l_{\alpha+n-1}+(n-\alpha-1) 3 e_{1} l_{\alpha+\beta+1}+ \\
& (n-\alpha-2)\left(e_{1}-e_{2}\right)\left(e_{1}-e_{3}\right) l_{\alpha+n+3} .
\end{aligned}
$$

The algebraic-geometric background for further degenerations has been studied in detail by one of us [19]. Note for instance that by $e_{1}=e_{2}=e_{3}=0$ this algebra becomes a representation for the centerless Virasoro algebra.

\section{Central extensions by $b-c$ systems}

Such central extensions in general for two point cases were studied in [1]. The properties of $b-c$ systems have been generalized to more than two punctures $[17,18]$. Explicit expressions for our case of two points had been obtained by the formula given in [1]. In this last section we investigate some aspects of these generalizations to the algebra (21)-(23). In order to get expressions which are easier to compare with the centrally extended Virasoro algebra we perform an index shift $e_{i}:=l_{i+1}$ and denote by $C_{i j}^{k}$ the structure constants of $\left[e_{i}, e_{j}\right]$.

By integrating the product of a $\lambda$-form and a $(1-\lambda)$-form over a nonsingular level line $C_{u}$ a dual pairing is introduced. It does not depend on $u \in \mathbb{R}$ (see [17] for details). Here we consider only $(\lambda, 1-\lambda)=(-1,2)$.

Proposition. The objects $\Omega_{k}:=A_{-k-2}(d z)^{2}$ and $e_{j}$ with $k, j \in \mathbb{Z}$ form a dual system by the product

$$
\langle e, \Omega\rangle:=\frac{1}{2 \pi \mathbf{i}} \oint_{C_{u}} e \cdot \Omega .
$$


Proof. Because all curves $C_{u}$ are homologous one can use local residue calculus around $P_{1}$ as well as around $Q_{1}$ and $Q_{2}$. These two integrations (residue calculus) give two conditions for the non-vanishing of $\left\langle e_{j}, \Omega^{k}\right\rangle$ which taken together yield

$$
\mathrm{i}\left(e_{j}\right)\left(\Omega_{k}\right):=\left\langle e_{j}, \Omega^{k}\right\rangle=c_{k} \delta_{i}^{k} .
$$

$c_{k}=1$ follows from the exact calculation of the residue at $P_{1}$.

Let $V$ be the vector space generated by all semi-infinite forms of weight 2

$$
\Omega^{i_{1}} \wedge \Omega^{i_{2}} \wedge \cdots \wedge \Omega^{s} \wedge \Omega^{s-1} \wedge \Omega^{s-2} \wedge \cdots
$$

with $i_{1}>i_{2}>\ldots s>s-1 \ldots$ and where the sequence of $\Omega^{i}$ contains all indices smaller than $s$ (for some $s$ which is not fixed). Furthermore we have operators $c^{i}, b_{k}$ defined by $c^{i}:=\Omega^{i} \wedge$ and $b_{k}:=\mathrm{i}\left(e_{k}\right)+$ product rule (anticommuting). As one can verify these operators satisfy a Clifford algebra

$$
\left\{b_{k}, c^{i}\right\}:=b_{k} \circ c^{i}+c^{i} \circ b_{k}=\delta_{k}^{i}, \quad\left\{b_{k}, b_{l}\right\}=\left\{c^{i}, c^{j}\right\}=0 .
$$

The following results are a concrete application of [1] and [18]. The vacuum vector $|0\rangle:=\Omega^{-2} \wedge \Omega^{-3} \wedge \Omega^{-4} \wedge \cdots$ has the properties

$$
c^{i}|0\rangle=0, \quad i<-1 \quad \text { and } \quad b_{k}|0\rangle=0, \quad k \geq-1 .
$$

We get a normal ordering by $: b_{k} c^{i}::=b_{k} c^{i}$ for $i<-1$ and $-c^{i} b_{k}$ for $i \geq-1$.

For general $b-c$ systems of weight $(\lambda, 1-\lambda)$ the energy momentum tensor is defined by $[18]$

$$
T(z):=:(1-\lambda) c(z) \partial_{z} b(z)-\lambda\left(\partial_{z} c(z)\right) b(z): .
$$

$T$ is a form of weight 2 and can be expanded as $T(z)=\sum_{k} L_{k} \Omega^{k}(z)$. The operator valued coefficents are

$$
L_{i}=\frac{1}{2 \pi \mathbf{i}} \oint_{C_{u}} T \cdot e_{i}
$$

by the duality (27). Here we have $\lambda=2$. Using the expansion $c(z)=\sum_{i} c^{i} e_{i}(z)$, $b(z)=\sum_{j} b_{j} \Omega^{j}(z)$ and recognizing (30) essentially as the Lie derivative [18]

$$
L_{c}\left(g_{\lambda}\right)=c \partial_{z} g_{\lambda}+\lambda g_{\lambda} \partial_{z} c \quad\left(g_{\lambda} \text { a } \lambda \text {-form }\right)
$$

we finally obtain the result

$$
L_{i}=\sum_{j, k} C_{i j}^{k}: b_{k} c^{j}:
$$


By explicit calculation one can see that these elements satisfy a centrally extended KN algebra for the singularity set $S$ and cause the relation

$$
\left[L_{i}, L_{j}\right]=-\sum_{k} C_{i j}^{k} L_{k}+\chi_{i j} \quad \text { with } \quad \chi_{i j}=\left(\sum_{A}-\sum_{B}\right) C_{i k}^{l} C_{j l}^{k} .
$$

The sets $A$ and $B$ of double indices are given by $A:=\{k<-1, l \geq-1\}$, $B:=\{k \geq-1, l<-1\}$. It is worthwhile noting that this formula (31) is independent from the number of markings. The only fact which is important with respect to this kind of representations is the generalized grading of the algebra. This grading is responsible for the $L_{i}$ to be well defined operators on $V$ because then $L_{i}$. (basis vector) always is a finite sum.

Let us give the cocycle and show its formal deformation to the cocycle of the Virasoro algebra. By direct but tedious calculations one obtains the following results (see [13] for details): First we get

$$
\chi_{\alpha n}=\chi_{n \alpha}=0 \quad \text { for } \quad n, \alpha+1 \in 2 \mathbb{Z} .
$$

To write down the remaining terms we use the following abbreviations: $I:=\{4,5,6,7\}$, $\beta_{i}:=\beta+i, m_{i}=m+i$ and

$$
\begin{gathered}
\lambda_{4}:=1, \quad \lambda_{5}:=3 \wp(1 / 2+q), \\
\lambda_{6}:=3 \wp^{2}(1 / 2+q)-\left(e_{2}^{2}+e_{2} e_{3}+e_{3}^{2}\right), \quad \lambda_{7}:=1 / 4 \wp^{\prime 2}(1 / 2+q)
\end{gathered}
$$

for the constants coming from the structure constants $C_{k l}^{r}$. In addition we define

$$
Q_{k}:=\sum_{i, j \in I} \lambda_{i} \lambda_{j} \delta_{i \cdot j}^{k}
$$

With this we receive (see [13])

$$
\begin{array}{rl}
\chi_{\alpha \beta}= & 13 / 6\left(\beta^{3}-\beta\right) \delta_{\alpha+\beta}^{0}+\left(13 / 6 \beta_{1}^{3}-2 / 3 \beta_{1}\right) \delta_{\alpha+\beta}^{-2} Q_{20}+ \\
& \left(13 / 6 \beta_{2}^{3}-25 / 6 \beta_{2}\right) \delta_{\alpha+\beta}^{-4} Q_{24}+\left(13 / 6 \beta_{3}^{3}-76 / 6 \beta_{3}\right) \delta_{\alpha+\beta}^{-6} Q_{28}^{*}, \\
\chi_{n m}=1 & 13 / 6\left(m^{3}-m\right) \delta_{m+n}^{0}+\left(13 / 3 m_{1}^{3}+5 / 3 m_{1}\right) \delta_{m+n}^{-2} Q_{20}+ \\
& \left(13 / 3 m_{2}^{3}+11 / 3 m_{2}\right) \delta_{m+n}^{-4} Q_{24}+\left(13 / 6 m_{2}^{3}-2 / 3 m_{2}\right) \delta_{m+n}^{-4} Q_{25}+ \\
& \left(13 / 3 m_{3}^{3}+5 / 3 m_{3}\right) \delta_{m+n}^{-6} Q_{28}^{*}+\left(13 / 3 m_{3}^{3}-25 / 3 m_{3}\right) \delta_{m+n}^{-6} Q_{30}+ \\
& \left(13 / 3 m_{4}^{3}-58 / 3 m_{4}\right) \delta_{m+n}^{-8} Q_{35}^{*}+\left(13 / 6 m_{4}^{3}-73 / 6 m_{4}\right) \delta_{m+n}^{-8} Q_{36}+ \\
& \left(13 / 3 m_{5}^{3}-133 / 3 m_{5}\right) \delta_{m+n}^{-10} Q_{42}^{*}+\left(13 / 6 m_{6}^{3}-110 / 3 m_{6}\right) \delta_{m+n}^{-12} Q_{49}^{*} .
\end{array}
$$


Here we have denoted all those $Q_{k}$ with a star which will vanish for $q=0$.

In a first step we degenerate to the two point case. Because the structure constants depend continuously on $q$ and because they reduce to those in the two-point case if $q$ equals zero we necessarily get the cocycles of the two-point case. In a second step we degenerate to $e_{1}=e_{2}=e_{3}=0$ and find that all remaining terms in which $Q_{k}$ occur become zero. What remains ist the well known extension form the Witt algebra to the Virasoro algebra

$$
\left[L_{m}, L_{n}\right]=(m-n) L_{m+n}+\frac{13}{6}\left(m^{3}-m\right) \delta_{m+n}^{0}, \quad m, n \in \mathbb{Z} .
$$

This expected result is a further motivation for the research on degenerated tori.

\section{Acknowledgements}

The first author (A.R.) likes to thank R. Dick for very fruitful discussions.

\section{REFERENCES}

1. Bonora, L., Matone M. Rinaldi, M., Relations between representations of KN and Virasoro algebras, Phys. Lett. B 216 (1989), 313.

2. Deck, Th., Stringtheorie und Krichever-Novikov-Algebren auf Tori, Diplomarbeit Universität Karlsruhe 1990.

3. Deck, Th., Deformations from Virasoro to Krichever-Novikov algebras, Phys. Lett. B 251 (1990), $535-540$.

4. Dick, R., Krichever-Novikov-like bases on punctured Riemann surfaces, Lett. Math. Phys. 18 (1989), 255.

5. Dick, R., Global expansions of holomorphic differentials on punctured Riemann surfaces, desy preprint 89-160 (1989).

6. Dick, R., Chiral fields on Riemann surfaces and string vertices, Dissertation Universität Hamburg, Germany 1990.

7. Dick, R., Topological Aspects of chiral fields in two dimensions and superstring vertices, Fortschritte der Physik.

8. Dick, R., The quadruplet of central charges on the sphere, Proceeedings of the $14^{\text {th }}$ John Hopkins workshop on current problems in particle theory (1990), eds. Domokos, G., Kövesi-Domokos, S..

9. Guo, H-y., Na, J-s., Shen, J-m., Wang, S-k., Yu, Q-h., The algebras of meromorphic vector fields....., Commun. Theor. Phys. 12 (1989), 349-354.

10. Hurwitz A., Courant R., Allgemeine Funktionentheorie und elliptische Funktionen, Springer, 1964.

11. Krichever, I.M., Novikov, S.P., Algebras of Virasoro type, Riemann surfaces and structures of the theory of solitons, Funktional Anal. i. Prilozhen. 21 (1987), no. 2, 46; Virasoro type algebras, Riemann surfaces and strings in Minkowski space, Funktional Anal. i. Prilozhen. 21 (1987), no. 4, 47.

12. Rauch, H.E., Lebowitz, A., Elliptic functions, theta functions and Riemann surfaces, Williams \& Wilkins, Baltimore, 1973. 
13. Ruffing, A., Prozessinduzierte symmetrische Basiskonstruktion ...., Diplomarbeit Universität Karlsruhe 1991.

14. Schlichenmaier, M., An introduction to Riemann surfaces, algebraic curves and moduli spaces, Lecture Notes in Physics 322, Springer, 1989.

15. Schlichenmaier, M., Krichever-Novikov algebras for more than two points, Lett. Math. Phys. 19 (1990), $151-165$.

16. Schlichenmaier, M., Krichever-Novikov algebras for more than two points: explicit generators, Lett. Math. Phys. 19 (1990), 327-336.

17. Schlichenmaier, M., Central extensions and semi-infinite wedge representations of KricheverNovikov algebras for more than two points, Lett. Math. Phys. 20 (1990), 33-46.

18. Schlichenmaier, M., Verallgemeinerte Krichever - Novikov Algebren und deren Darstellungen, Dissertation Universität Mannheim , Germany(1990).

19. Schlichenmaier, M., Degenerations of generalized Krichever-Novikov algebras, Mannheimer Manuskripte 137 (1992). 